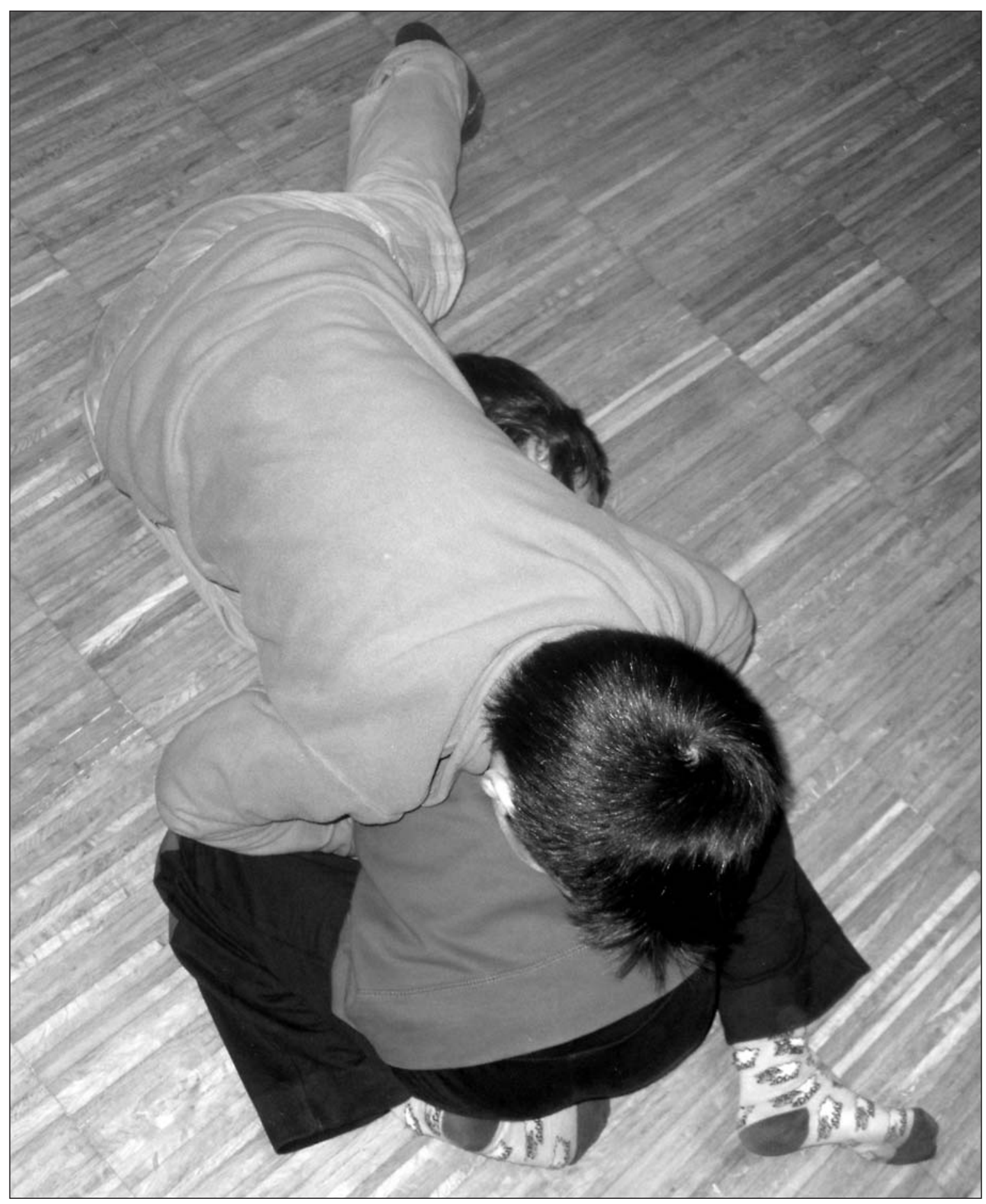

\title{
Resumen
}

El presente estudio, de carácter teórico, tiene por objeto exponer las líneas fundamentales de un modelo didáctico para la iniciación a los deportes de combate. Para ello se analizan las aportaciones de numerosos autores que desde diversas perspectivas consideran que la iniciación a los deportes de combate no significa iniciarse específicamente en alguna de sus modalidades, sino que implica un tratamiento integrado y transversal de las mismas. Estas concepciones "alternativas" restan importancia a los modelos técnicos específicos para potenciar los elementos comunes de los deportes de combate, y particularmente el desa-

Fotografía cortesía de Bruno Avelar rrollo de lo que ha venido a denominarse el "saber luchar"; esto es, la capacidad que permite al luchador solucionar las diversas situaciones que se suceden durante un combate. 


\section{LA INICIACIÓN A LOS DEPORTES DE COMBATE: INTERPRETACIÓN \\ DE LA ESTRUCTURA DEL FENÓMENO LÚDICO LUCTATORIO}

Bruno Avelar \& Abel Figueiredo

\section{Introducción}

Una de las líneas de investigación científica de las artes marciales y deportes de combate que ha experimentado una mayor producción durante las últimas décadas es la relacionada con los procesos de enseñanza y aprendizaje de estas disciplinas (e.g., véase para el caso español Gutiérrez \& Pérez, 2009). Particularmente, la enseñanza mediante modelos eclécticos y globales viene siendo objeto de reflexión y desarrollo desde la segunda mitad de los años 80 (Terrisse, 1996; Espartero, Gutiérrez \& Villamón, 2003). No obstante, existe una diferencia significativa entre la producción referida a los deportes de combate con agarre, mucho más abundante, y la relativa a los deportes de combate de golpeo, con una producción mucho menor ${ }^{1}$.

En este sentido, actualmente ya "son numerosos los autores que, partiendo de una iniciación genérica y multidisciplinar, han postulado a favor de una iniciación polideportiva en la que se adquieran y perfeccionen toda las habilidades propias de las actividades de lucha" (Espartero, Gutiérrez \& Villamón, 2003:301). Así, Figueiredo (1998:I) señala que iniciar una formación en deportes de combate "no es iniciarse en 'Lucha', 'Judo', 'Boxeo', 'Esgrima', 'Kárate', etc.”, y en la misma línea Lima (1997:19) afirma que el tratamiento de los deportes de combate "apunta hacia una formación ecléctica, donde los contenidos y materias deben de estar sujetos a las exigencias del éxito del proceso de enseñanza-aprendizaje, debiendo los Deportes de Combate ser considerados de forma integrada". No obstante, parece que la realidad camina por diferentes derroteros, incluso en un ámbito tan global como es el de la educación física escolar. Así, Figueiredo (1997, 1998, 2008) en Portugal, tras analizar los programas curriculares de la materia, señala que en los mismos se defiende una alternativa más bien introductoria a modalidades deportivas concretas que una iniciación multidisciplinar.

Según los planteamientos globales, no se trata de proponer una iniciación específica a una o varias modalidades de deportes de lucha o combate, sino observar e integrar el conjunto de elementos comunes que las caracterizan. De este modo, el proceso de enseñanza-aprendizaje se centrará más bien en el planteamiento de las tareas -cuya 
estructuración juega un papel fundamental-, que en las técnicas particulares de cada disciplina en cuestión (proyecciones, controles, golpes de pierna o de puño, etc.).

Como se podrá observar en este artículo, esta perspectiva globalizadora incide en la dinámica del fenómeno luctatorio, donde la distancia determina el tipo de acción a realizar y cuyo enfoque, basado en el desarrollo de los aspectos perceptivos, permite promover lo que ha venido a denominarse el "saber luchar" (Terrisse, 1991, 1996; Terrisse y cols., 1995). Entendemos el saber luchar como aquella capacidad que permite al deportista solucionar las diversas situaciones que se suceden durante un combate, independientemente de los modelos técnicos de ejecución que caracterizan las diferentes modalidades y cuyo reconocimiento institucional impone determinadas restricciones.

Seguidamente, se presentará la construcción teórica que justifica y da soporte a la iniciación a los deportes de combate bajo esta perspectiva global y horizontal. Dicha construcción se basa en los denominados Modelos Comprensivos o Alternativos de la Iniciación Deportiva, desarrollados a partir del modelo anglosajón Teaching Games for Understanding ${ }^{2}$. También consideramos el trabajo de diversos autores ibéricos ${ }^{3}$ que utilizan los fundamentos de la Praxiología Motriz como eje de observación de la realidad luctatoria, e igualmente el trabajo de algunos autores franceses ${ }^{4}$ que basan su análisis en el conocimiento desarrollado en la dinámica de combate.

Nuestro objetivo con este planteamiento teórico es que pueda servir de base para la aplicación de un proyecto empírico que permita observar la dimensión real del proceso de enseñanza-aprendizaje de los deportes de combate en su sentido global. Como afirman Espartero, Gutiérrez \& Villamón (2003:32), "si bien son muchos los autores que se han preocupado de describir y fundamentar los objetivos educativos (a nivel físico, psicomotor, social, ético, etc.) de las actividades de lucha, la valoración empírica acerca de la consecución de los mismos es prácticamente inexistente".

\section{La estructura de los deportes de combate y el desarrollo del "saber luchar"}

En la caracterización de los deportes de combate, entendidos globalmente, la "distancia de enfrentamiento motor" asume un papel central, una vez que "determinada por las reglas, esta distancia caracteriza cada tipo de confrontación” (Parlebas, 1988:136). Asimismo, las interacciones en contacto, en el espacio próximo y en el espacio medio, determinan la denominada "distancia de guardia", la cual representa la "distancia de enfrentamiento motor que separa a dos adversarios en el momento inmediatamente anterior a la acción de uno de ellos" (Parlebas, 2001:152). Tomemos, por ejemplo, la distancia que mantienen los practicantes al tratar de agarrar a su adversario en judo (distancia corta) o al tratar de realizar un golpe en kárate (distancia media): la distancia de guardia desde la que se parte en ambas situaciones es diferente y determina el éxito de la acción motriz posterior.

Los espacios de interacción motriz son el criterio clave en la determinación del tipo de acción motriz y de los respectivos roles en la práctica de combate. En este sentido, Amador (1997:57) señala que "la utilización del espacio de lucha puede presentar diferentes características que tienen relación, entre otras, con la propia finalidad de la lucha". De hecho, según el tipo de interacción que se produzca, la utilización del espacio será diferente, así como las propias características del espacio en el que ocurren las prácticas de combate institucionalizadas (por ejemplo, el área de combate reglamentaria en judo, kárate o lucha libre).

Asimismo, la distancia y, en relación a la misma, la utilización del espacio, determinan la acción motriz a realizar por ambos practicantes; así, al espacio próximo le

La iniciación a los deportes de combate... $\diamond$ Bruno Avelar \& Abel Figueiredo 
corresponden unas acciones motrices determinadas, mientras que las interacciones en el espacio lejano implican la utilización de otro tipo de acciones (véase la Figura 1).

\begin{tabular}{|c|c|c|}
\hline Larga Distancia & Media Distancia & Corta Distancia \\
\hline $\begin{array}{l}\text { GOLPEOS CON } \\
\text { IMPLEMENTOS }\end{array}$ & $\begin{array}{c}\text { GOLPEOS } \\
\text { CON LAS } \\
\text { EXTREMIDADES }\end{array}$ & GOLPEOS Y AGARRES \\
\hline \multicolumn{2}{|c|}{ Empuñar, Golpear, Tocar, Parar, Bloquear, Esquivar } & $\begin{array}{c}\text { Tirar, Empujar, Inmovilizar, Proyectar/ } \\
\text { Derribar, Volcar, Caer, Fijar, Excluir, } \\
\text { Controlar, Tocar, Golpear }\end{array}$ \\
\hline
\end{tabular}

Figura 1: Tipos de interacción y acciones motrices según la distancia de enfrentamiento.

Desde esta perspectiva de utilización de la acción motriz como criterio de diferenciación entre las prácticas de combate, quizá sea Guillermo Torres $(1989,1990)$ quien mejor haya mostrado una concepción de base más profunda respecto al entendimiento global de estas prácticas, destacando las acciones motrices que derivan de las distintas distancias de enfrentamiento. Torres organiza las habilidades motrices propias de las luchas en grupos taxonómicos que denomina "unidades motrices básicas", que son "aquellos actos motrices que han perdido su componente técnico-deportivo y conservan su potencial educativo" (Torres, 1990:47). A estas unidades motrices básicas corresponden las "unidades motrices primarias" (agarrar y golpear), en función de la mayor o menor distancia a la que se encuentran ambos practicantes. A partir de las unidades motrices primarias se generan las "unidades motrices secundarias" (tirar, empujar, inmovilizar, proyectar/derribar, volcar, caer, tocar, empuñar, parar, esquivar), que representan las acciones luctatorias que sirven de base genérica al futuro desarrollo de técnicas específicas de combate.

Las técnicas específicas de combate deben ser consideradas en función de lo que Figueiredo (1998) llama "situaciones de combate" (golpeos/toques, proyecciones/derribos, inmovilizaciones, luxaciones y estrangulaciones), y representan las acciones esenciales de finalización que diferencian las prácticas de lucha, y cuya estructura incluye las unidades motrices primarias y secundarias. Con mayor o menor énfasis en algunos de sus elementos, las disciplinas constituyentes de los deportes de combate presentan un hibridismo considerable respecto a la utilización de dichas situaciones de combate en su repertorio técnico, dependiendo su aplicación de circunstancias como la ya señalada distancia entre ambos contrincantes.

Asimismo, la distancia de enfrentamiento estimula, en primer lugar, el tipo de confrontación entre los contrincantes (agarre o golpeo), y también la acción motriz que cada uno de ellos puede realizar. En relación a esto, la "distancia de guardia entre los adversarios concebida bidimensionalmente, es primordial, ya que impone para cada competidor su estatus motriz" (Amador, 1997:356), el cual representa el rol (ofensivo o defensivo) que un practicante asume en un determinado momento del combate.

Para Figueiredo (1998:III) es la percepción de la distancia por parte de los protagonistas en situación lúdica de combate lo que les permite decidir qué ritmo y rol deberá ser asumido, surgiendo los desplazamientos como acciones determinantes en la ruptura de las distancias.

En relación a los roles durante el combate, Castarlenas (1993) describe así la estructura y dinámica fundamental de roles durante el combate (véase la Figura 2): 


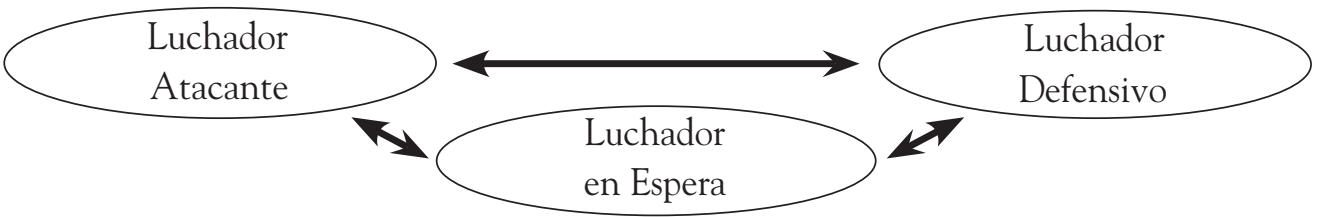

Figura 2: Estructura y dinámica fundamental de roles (en Castarlenas, 1993:61).

A los roles ofensivo y defensivo se asocian diferentes objetivos que deben ser considerados desde el punto de vista estratégico según el tipo de enfrentamiento. Estos objetivos son señalados por Molina \& Villamón (2000:179) y Molina \& Castarlenas (2002:35) (véase la Figura 3).

\begin{tabular}{|c|c|c|}
$\begin{array}{c}\text { Grupo de Deporte } \\
\text { de Combate }\end{array}$ & $\begin{array}{c}\text { Principios Estratégico- } \\
\text { Tácticos de Ataque }\end{array}$ & $\begin{array}{c}\text { Principios Estratégico- } \\
\text { Tácticos de Defensa }\end{array}$ \\
\hline Deporte de Combate con Agarre & $\begin{array}{c}\text { Proyectar o controlar } \\
\text { al adversario }\end{array}$ & $\begin{array}{c}\text { Evitar ser proyectado o } \\
\text { controlado por el adversario }\end{array}$ \\
\hline Deporte de Combate con Golpeo & $\begin{array}{c}\text { Hacer blanco en el cuerpo } \\
\text { del adversario }\end{array}$ & $\begin{array}{c}\text { Evitar ser golpeado por } \\
\text { el adversario }\end{array}$ \\
\hline Deporte de Combate con Armas & $\begin{array}{c}\text { Hacer blanco en el cuerpo } \\
\text { del adversario }\end{array}$ & $\begin{array}{c}\text { Evitar ser tocado por el arma } \\
\text { del adversario }\end{array}$ \\
\hline
\end{tabular}

Figura 3: Principios estratégico-tácticos según los diferentes tipos de Deportes de Combate.

El interés de estos objetivos, que conllevan diferentes principios estratégicotácticos, es que en los mismos subyacen cuestiones relacionadas con la intencionalidad del practicante, a través de la toma de "iniciativa" o "pasividad" por parte de alguno de estos (Molina \& Castarlenas, 2002:35), aunque evidentemente uno puede asumir un planteamiento defensivo activo, sin que esté en situación de pasividad (véase, por ejemplo, la utilización de una estrategia de contra-ataque o la doble funcionalidad de la guardia, la cual posibilita tanto la defensa como el ataque). Tal como afirma Figueiredo (2003:17), "la posición fundamental de transición (guardia) asume relevancia en cualquier acción técnica de ataque o de defensa, pues la fase de transición en que no tenemos fase ofensiva o defensiva es una fase clara de guardia".

En este sentido, consideramos que la estructura fundamental en esta dinámica de roles es la referente al "luchador en espera", pues esta representa la determinación de la distancia de guardia -asumiéndola, tal como hemos citado, como el momento inmediatamente anterior a la acción de uno de los luchadores- pero también la situación donde cada practicante toma la decisión respecto a la acción a ejecutar seguidamente, representando por ello, un "proceso de transición" que sucede entre la asunción o refuerzo de un rol ofensivo o defensivo. También debemos considerar que, debido a la evidente fluidez entre los roles de ataque y de defensa, esta situación de luchador en espera puede no llegar a producirse; tal es el caso, por ejemplo, de la realización de varios ataques ininterrumpidos. Asimismo, consideramos que este proceso de transición es dinámico, ya que, desde una perspectiva ecológica, la percepción, la decisión y la acción no se suceden sino que interactúan.

La existencia de un proceso de transición que permite asumir o reforzar un rol representa la posibilidad de que emerja una "intención táctica", la cual invoca "la necesidad de reducir la incertidumbre para facilitar el aprendizaje y al mismo tiempo conservar

La iniciación a los deportes de combate... $\diamond$ Bruno Avelar \& Abel Figueiredo 
las fases de confrontación sin que se pierda el sentido de la actividad" (Terrisse y cols., 1995:26-27). Para estos autores, la intención táctica representa el hecho de saber luchar y es el objetivo prioritario en la enseñanza de los deportes de combate, en cualquiera de sus variantes.

También podemos observar que la intención táctica desarrollada en el mencionado proceso de transición también está representada en los ejes "ganar-perder" y "atacardefender" destacados por Terrisse (1991) ${ }^{7}$. Por un lado, en el primer eje ganar-perder, la táctica consiste en provocar un fallo en el sistema defensivo del otro, sabiendo que esta intención es recíproca. Por otro lado, en el segundo eje atacar-defender, se puede observar que "todo ataque contiene, en efecto, los elementos de su fracaso pues exige una apertura de la guardia o la creación de un desequilibrio consecutivo a un desplazamiento. La guardia pierde su función de preservación de la neutralidad en la medida que condiciona a la vez tanto el ataque como la defensa. Su apertura es una condición previa a toda ofensiva pero contiene al mismo tiempo los gérmenes de la contra y, entonces, su propia negación" (Terrisse, 1994:11).

El mismo Terrisse (1996) señala que, actualmente, la evolución del rendimiento apunta hacia una lógica decisional que permita solucionar una situación a través de decisiones tomadas a partir de determinados estímulos (por ejemplo: si él... yo...). Así, el conocimiento de las diferentes posibilidades de actuación implica la reducción de la incertidumbre del practicante y el aumento de su "intención táctica"; es decir, la evolución de su noción de saber luchar. La construcción de posibles esquemas de actuaciones encuentra soporte en el trabajo de Collinet $(1992)^{8}$, quien plantea la construcción de algoritmos ${ }^{9}$. Los algoritmos son precisamente estos esquemas que describen las posibilidades de interacción a través de la mencionada interrelación de "si él... yo...", como por ejemplo el clásico "si él tira... yo empujo / yo le empujo y bloqueo su apoyo". Este autor considera, en el caso de los llamados deportes de combate con agarre, las alternativas que cada practicante puede asumir en situaciones determinadas, aunque reconozca que "la utilización y presentación de un algoritmo a los alumnos, no es suficiente para garantizar un aprendizaje eficaz" (Collinet, 1994:29). De hecho, dada la complejidad de la práctica y las diferentes capacidades de los practicantes, el conocer el abanico de posibilidades no será suficiente, pero permitirá al profesorado plantear diversas tareas en función de la lógica planteada en el algoritmo.

\section{El proceso de enseñanza-aprendizaje en los deportes de combate}

El proceso de enseñanza-aprendizaje en la iniciación a los deportes de combate desde una perspectiva global ha de promover "la construcción de situaciones de aprendizaje de estructuras, sino idénticas, al menos comparables" (Terrisse, 1994:9). Como hemos señalado, este planteamiento intenta superar la enseñanza específica de los diferentes deportes de combate, y se fundamenta en el análisis estructural realizado anteriormente. De hecho, "el recurso a las clasificaciones constituye un medio que permite reagruparlas, valorando en el seno de una misma familia lo que las reúne más que lo que las separa, (...) [poniendo] el acento sobre los caracteres comunes o permanentes de actividades que son próximas" (Terrisse, 1994:9).

Asimismo, diversos autore ${ }^{10}$ presentan propuestas de iniciación a los deportes de combate en su sentido integrado y según un modelo horizontal, aunque como se ha señalado ninguna se haya investigado empíricamente. Como nexos comunes, estas propuestas presentan un hilo conductor que va desde los juegos de cooperación/oposición inespecíficos hacia las situaciones de combate específicas de las diferentes disciplinas 
de lucha. Tal como afirma Brousse (1993:50), se trata de un proceso basado "en la progresión de la dificultad de los contenidos abordados (grado de oposición) y de la complejidad (jerarquización de las secuencias técnico-tácticas)”. En este sentido, Molina \& Castarlenas (2002:43) presentan un conjunto de directrices que consideramos relevantes en el proceso de iniciación a los deportes de combate, englobando la mayor parte de orientaciones que presentan los autores antes citados:

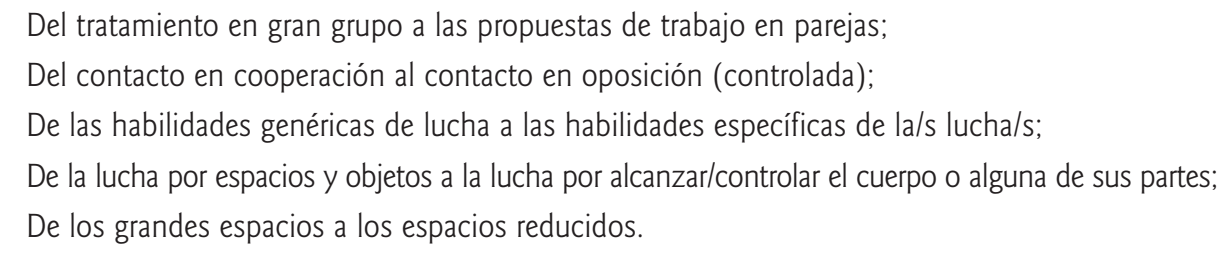

En cuanto a la metodología utilizada en el planteamiento de las tareas, Castarlenas (1990:27) señala que "creemos más adecuados los sistemas de enseñanza basados en la RESOLUCIÓN DE PROBLEMAS, ya que estos desvelan la creatividad y facilitan que las soluciones a las situaciones planteadas sean el fruto de un proceso de razonamiento". Bronchart (1994:7), en la misma línea, defiende la utilización de los métodos activos en la enseñanza de los deportes de combate, afirmando que es importante "incluir la lógica del combate en el estudio de una lógica psicomotriz de construcción y de búsqueda de gestos elaborados por el alumno en situaciones reales de oposición. Estos gestos vienen de materiales apropiados a su vivencia de la actividad". En este sentido, creemos que la actividad lúdica desarrollada a través de tareas abiertas es el eje central en la iniciación a los deportes de combate desde una perspectiva global, ya que más que centrarnos en la ejecución técnica de los gestos lo que nos importa, principalmente, es la dinámica (lúdica) de la lucha, a través de juegos que potencien el desarrollo del "saber luchar" (utilizando juegos de distancia, de toque, de desequilibrio, de inmovilización, de saludo). De hecho, tal como afirma Figueiredo (1998:II), "los objetivos del propio juego señalan la predominancia táctica sin olvidar indicadores técnicos importantes, es decir, además del correcto encuadramiento del espíritu lúdico-luctatorio asumido en el juego, son la gestión del factor táctico: buscar las informaciones pertinentes que sean esenciales para la elaboración de los planes que lleven a concretar esos objetivos". La mencionada gestión del factor táctico muestra una coincidencia casi total con la "intención táctica" propuesta por Terrisse y cols. (1995:26-27).

Sin embargo, a pesar de la importancia que otorgamos a la actividad lúdica y su relación con el desarrollo de la "intención táctica" antes citada, hay que señalar también la necesidad de un correcto aprendizaje de los gestos técnicos (primero genéricos y luego específicos), los cuales permitirán una mejor integración del practicante en las situaciones tácticas propuestas que suceden en la práctica agonística. Entre los dos extremos del hilo de la enseñanza deportiva (técnica y táctica) "se pueden plantear otras situaciones que permiten enfatizar un aspecto u otro" (López Ros \& Castejón, 1998b:12). Tal como afirman los mismos autores (ídem, 1998b:14), "el aprendizaje a través de la táctica, es decir, de la capacidad de resolver mentalmente problemas motores nos podría inclinar a utilizar, ante determinadas situaciones, la concienciación táctica sobre el perfeccionamiento de la ejecución motriz o técnica. Sin embargo, para poder llegar a ello, es necesaria una base motriz técnica mínima que permita poder ejecutar decisiones tácticas que se organizan sobre las habilidades motrices básicas". Se trataría, por tanto, de potenciar el desarrollo de la complejidad técnica a la vez que el desarrollo de la complejidad táctica,

La iniciación a los deportes de combate... $\diamond$ Bruno Avelar \& Abel Figueiredo 
sin olvidar ninguno de sus componentes ni su interrelación. De este modo, se incide en el desarrollo del "saber luchar" partiendo del esbozo del gesto situado en el contexto del juego sencillo, hasta la técnica específica practicada en situación agonística compleja, utilizando siempre las orientaciones lúdicas presentadas anteriormente como el principal eje evolutivo. Estas ideas sobre el acoplamiento de técnica y táctica llevan a López Ros \& Castejón (1998b:16) a proponer un modelo para la enseñanza de la técnica y la táctica en la iniciación deportiva (véase Figura 4).

\begin{tabular}{|l|c|}
\hline \multicolumn{2}{|c|}{ 1. Dominio de las habilidades y destrezas básicas. } \\
\hline $\begin{array}{c}\text { 2. Presentación de la táctica deportiva con } \\
\text { implicación de pocos elementos técnicos. }\end{array}$ & $\begin{array}{c}\text { 2. Presentación de la técnica deportiva con } \\
\text { implicación de pocos elementos tácticos. }\end{array}$ \\
\hline \begin{tabular}{|} 
3. Presentación de situaciones de juego similar al deporte definitivo con aplicación de los \\
elementos técnicos y tácticos aprendidos.
\end{tabular} \\
\hline $\begin{array}{l}\text { 4. Presentación de la táctica deportiva con } \\
\text { implicación de nuevos elementos técnicos. }\end{array}$ & $\begin{array}{c}\text { 4. Presentación de la técnica deportiva con } \\
\text { implicación de nuevos elementos tácticos. }\end{array}$ \\
\hline \multicolumn{2}{|c|}{ 5. Presentación de situaciones de juego similar al deporte definitivo con aplicación de los } \\
elementos técnicos y tácticos aprendidos.
\end{tabular}

Figura 4: Modelo para la enseñanza de la técnica y la táctica en la iniciación deportiva (López Ros \& Castejón, 1998b: 16).

Creemos, según lo expuesto, que este modelo puede representar una buena guía del proceso de enseñanza-aprendizaje en la iniciación a los deportes de combate, una vez que posibilita articular las "unidades motrices básicas" (gestos) señaladas por Torres $(1989,1990)$ con las "situaciones combate" (técnicas) propuestas por Figueiredo (1998) y también que, en su momento, ambas sean integradas en el desarrollo de la "intención táctica". Esta forma de trabajo habrá de tender al desarrollo de la capacidad de "saber luchar".

De un modo análogo, la gestión del acoplamiento cooperación/oposición es, desde nuestro punto de vista, otro punto clave para el manejo y aplicación de las tareas a utilizar en el proceso de enseñanza-aprendizaje. Siguiendo a Calmet \& Patinet (1993), la práctica de oposición puede entenderse según su potencial de cooperación, señalando las posibilidades y ventajas del aprendizaje de los deportes de combate a través de dos orientaciones de enseñanza: el "duelo" (en oposición-lucha) y el "dúo" (en cooperacióncoreografía). En este sentido, asumiendo el carácter simulado de las prácticas de algunas disciplinas de combate (las katas de las artes marciales japonesas, por ejemplo), presentan un trabajo en "cascada" donde el alumnado pasa por un proceso que, en una primera fase, es de cooperación y realizado bajo la forma de una coreografía, pero que después evoluciona hacia una práctica agonística de combate. Esta es una situación interesante debido al hecho de que la construcción del saber respecto a un mismo objeto, por haber sido aplicado en diferentes ámbitos, conlleva a distintas concepciones de ese mismo objeto valorando, por lo tanto, la permeabilidad de las actividades propuestas ${ }^{11}$, las cuales "permiten construir otros contenidos de enseñanza" (Calmet \& Patinet, 1993:67). Se puede relacionar esta perspectiva con los conceptos de "hacer contra" (oposición) y "hacer con" (cooperación) abordados posteriormente por el mismo Calmet (2000).

Asimismo, tal y como señala Terrisse (1994:14) "el trabajo propuesto comienza bajo la forma de una cooperación en que los papeles son definidos de antemano y en que la incertidumbre que viene del otro es prácticamente nula", lo que representaría 
una oposición controlada o simulada. De aquí se pasaría a una situación de ruptura de la prescripción, dotando la actividad de un carácter abierto desde el punto de vista de los roles a asumir, llevando a los practicantes hacia lo que este mismo autor llama "oposición dispuesta". Concluyendo por tanto que "la metodología de construcción de situacionesproblema en deportes de combate se apoya sobre la gestión de la unión dúo/duelo" (ídem). Es en este sentido que Figueiredo (1998:IV-VIII) asume en sus "situaciones características" la ejercitación de la oposición a través de la aplicación de diferentes roles cooperativos de "más bien atacante" y de "más bien defensor" en la realización de tareas con carácter lúdico (juegos de distancia, juegos de toques, juegos de desequilibrio, juegos de inmovilización, juegos de saludo) ${ }^{12}$, además de la posibilidad de determinar previamente quién ataca y quién defiende. De este modo se desarrolla el sentido de oposición a través de roles cooperativos sin que por ello se pierda el fundamento táctico agonístico que representa la esencia de la tarea. Es lo que Prieto y cols. (2001) han denominado "oposición cooperativa", que además de manifestarse en el propio desarrollo de los juegos y situaciones se refleja en toda la práctica a través de situaciones educativas como el "diálogo en los momentos de actividad no motriz, ayudas en la ejecución, fijación en las reglas establecidas para la actividad, aportación de ideas, aceptación del papel asignado, implicación en la creación de espacio, correcciones en las ejecuciones, demostraciones de ejecuciones, etc." (ídem).

\section{Conclusión}

Este trabajo recoge algunas de las aportaciones teóricas realizadas sobre la iniciación a los deportes de combate bajo una perspectiva integrada y según un modelo de tratamiento didáctico con carácter horizontal. Este modelo no supone una suma entre las diferentes disciplinas constituyentes de este grupo de deportes, sino un entendimiento amplio del fenómeno de combate, donde la distancia en que intervienen los practicantes es el eje que determina la acción a desarrollar por ambos contrincantes. Por otro lado, la intencionalidad que estos demuestran revela el desarrollo de su saber luchar, independientemente de las especificidades técnicas asociadas a las diferentes disciplinas de combate. Efectivamente, en todas las disciplinas de combate nos encontramos con una misma problemática (combate) utilizando diferentes herramientas (técnicas), expresadas según diferentes circunstancias (distancia, desplazamientos, contacto, referentes espaciales piesuelo, etc.).

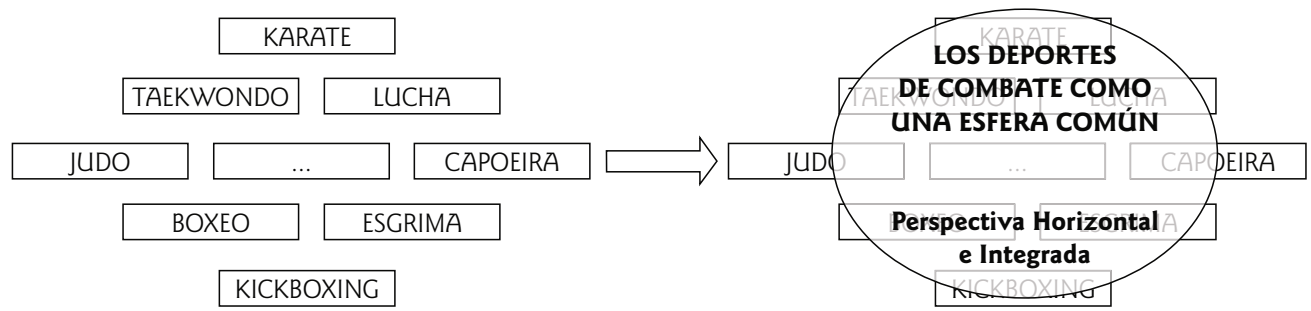

Figura 5: Planteamiento horizontal en la iniciación a los deportes de combate.

En este sentido, el planteamiento horizontal de la iniciación a los deportes de combate se asume como la base de un proceso de enseñanza-aprendizaje de carácter global. Dicha metodología debería potenciar la construcción de un repertorio amplio y consistente de habilidades genéricas que sirva de base a la posterior adquisición de las habilidades específicas, incluyendo asimismo el desarrollo de los aspectos perceptivos y 
decisionales de la acción. En la consecución de este proceso, que considera el "modelo para la enseñanza de la técnica y de la táctica en iniciación deportiva” (López Ros \& Castejón, 1998b), el tipo de tareas planteadas y su relación con los objetivos programados juegan un papel fundamental (véase la Figura 4.). De este modo, los juegos de lucha (juegos de distancia, de toques, de desequilibrio, de inmovilización, de saludo), son instrumentos fundamentales en el potencial desarrollo de situaciones de finalización de combate (golpeos/toques, proyecciones/derribos, inmovilizaciones, luxaciones y estrangulaciones), que representan las tareas esenciales y diferenciadoras de la práctica luctatoria ${ }^{13}$.

La perspectiva de iniciación a los deportes de combate que se ha presentado a lo largo de este documento está íntimamente relacionada con el planteamiento de la educación física escolar. Sin embargo, según Theebom \& Knop (1999) esta perspectiva, basada en un modelo comprensivo con carácter horizontal, no se manifiesta en la gran mayoría de los currículos europeos, los cuales inciden en el aprendizaje y desarrollo de modalidades de combate específicas. Por el contrario, lo que aquí planteamos es que este concepto de iniciación integrada puede implementarse no sólo en un ámbito escolar, sino también en otros ámbitos y en cualquier modalidad de deporte de combate, ya que esta es una perspectiva que posibilita el desarrollo de aprendizajes plásticos con capacidad de adaptación a las más diferentes situaciones luctatorias, independientemente de su especificidad.

En otro sentido, es importante señalar que este documento está centrado únicamente en el análisis de la estructura de los deportes de combate, el conocimiento de su dinámica y el proceso de enseñanza-aprendizaje derivado del mismo. Con todo, aunque no sea objeto de este trabajo, no debemos olvidar el potencial educativo de estas prácticas en relación a aspectos tales como la responsabilidad ante el otro a través del control de la agresividad, el aumento de la auto-estima, etc. Como afirma Olivera (1984:8) "la lucha representa para el Hombre, el volver a sus raíces, recuperando una serie de experiencias motrices básicas y decisivas en el conjunto de la motricidad humana. Desde el punto de vista educativo, es necesario por tanto, reivindicar la necesidad de contemplar en los programas de Educación Física, las actividades de lucha, partiendo desde unas posiciones etnomotrices, donde las actividades luctatorias entroncan con los Deportes de Combate". Asimismo, consideramos que "las actividades de lucha no son en ningún momento acciones motrices generadoras de violencia, sino que por el contrario, demuestran que a través de ellas es posible conquistar otras alternativas educativas" (Vergara, 2001:100).

De esta manera, creemos que una concepción y una organización didáctica basada en el proceso expuesto facilitaría que la reivindicación expuesta por Olivera fuese más consistente, al ir al encuentro de una educación física ecléctica e inclusiva (no tecnicista), tan pretendida por los más diversos autores y sistemas educativos. Sin embargo, seguimos carentes de aportaciones y valoraciones empíricas que determinen, efectivamente, cual es la dimensión de su potencial. Desde nuestro punto de vista, esta es una cuestión primordial y que pretendemos llevar a cabo en próximas investigaciones, asumiendo también el interés de una organización didáctica vertical según los niveles de desarrollo y madurez de los implicados como uno de los principales centros de interés.

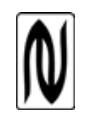

Revista de Artes Marciales Asiáticas $\diamond$ Volumen 4 Número 3 (44-57) - 2009 


\section{AGRADECIMIENTOS}

Los autores desean expresar su agradecimiento a la Associació Esportiva Ciutat Vella y a la comunidad escolar del CEIP Parc de la Ciutadella (ambas en Barcelona) por permitirnos realizar la fotografía de portada, y a Rodrigo Gonçalves y Cátia Coelho por la realización y tratamiento digital de la misma.

\section{NOTAS}

1 La diferenciación entre deportes de combate de agarre y deportes de combate de golpeo tiene un carácter meramente circunstancial una vez que, por cuestiones prácticas y didácticas, resulta sencillo agrupar las diferentes disciplinas de combate en estos grupos. Sin embargo, somos conscientes que prácticamente todas las disciplinas de combate presentan un hibridismo entre estos grandes grupos. Por otro lado, nuestra posición se basa en que la percepción de las distancias cambiantes determina considerablemente el tipo de interacción, que será consecuencia del tipo de tareas planteadas y no de las especificidades técnicas eventualmente consideradas.

2 El modelo Teaching Games for Understanding (TGfU) es una teoría propuesta por Bunker \& Thorpe (1982) cuyo modelo sirve de base a los modelos comprensivos de enseñanza. Se trata de una teoría que basa sus principios en el desarrollo de las habilidades deportivas según las necesidades impuestas por el entorno (habitualmente el juego). Las técnicas son aprendidas y entrenadas según sus posibilidades y necesidad de aplicación en la realidad de dicho entorno. En su planteamiento didáctico, el TGfU supone la integración del alumno en el juego, donde puede hacer su apreciación y adquirir la conciencia táctica que permitirá la toma de decisiones y consecuente ejecución técnica. En este modelo resulta especialmente relevante la aplicación de conceptos como transferencia (intra e intertareas), o la exageración y representación de situaciones de juego. Por tanto, la táctica, es decir, la compleja dinámica del juego, es el eje de aprendizaje y desarrollo de los alumnos. El TGfU se ha desarrollado de manera más marcada en los deportes colectivos.

3 Entre ellos destacamos a Torres (1984, 1989 \& 1990), Hernández Moreno (1985), Garcia Fojeda \& Castarlenas (1988), Castarlenas (1990), Lima (1990, 1995 \& 1997), Carratalà (1990, 1998), Amador (1995, 1996, 1997), Simon (1997), Figueiredo $(1997,1998)$ y Espartero, Gutiérrez \& Villamón (2003).

4 Entre ellos destacamos a Terrisse (1991), Collinet (1992), Brousse (1993), Calmet \& Patinet (1993), Bronchart (1994), Terrisse y cols. (1995) y Terrisse (1996).

5 Parlebas (1988:136) define la "distancia de enfrentamiento motor" como "el valor medio de la distancia que separa a dos adversarios en el momento de enfrentamiento directo".

6 La designación original se refiere a una "Intention stratégique". Sin embargo, debido al concepto que expresan los autores bajo esta denominación, creemos que es más adecuado hablar de táctica (cómo solucionar los problemas observados durante el combate) que de estrategia (la cual se refiere al planteamiento previo de la dinámica del combate), pues tal como señalan López Ros \& Castejón (1998a:6) la estrategia se refiere a "las normas de funcionamiento establecidas antes de la práctica motriz". En relación a estos aspectos, el trabajar las situaciones tácticas es trabajar en la franja de información susceptible de ser captada por el practicante, es decir, se le da la posibilidad de toma de conocimiento del contexto agonístico donde está implicado, pues se considera que la información no está en el practicante sino en el contexto de práctica (Araújo \& Volossovitch, 2005).

La iniciación a los deportes de combate... $\diamond$ Bruno Avelar \& Abel Figueiredo 
7 Terrisse, A. (1991). "Pour un enseignemenent dialectique des sports de combat". Revue E.PS, 229, 23-26. / Terrisse, A. (1994). "Para una enseñanza 'Dialéctica'“. Revista de Educación Física, 59, 9-14. Nos basamos en la versión española del artículo.

8 Collinet, S. (1992). "Algorithme et EPS. Un Exemple: Les Sports de Combat de Préhension“. Revue E.PS, 237, 25-28. / Collinet, S. (1994). "Algoritmo y Educación Física-Deportiva. Un Ejemplo: los Deportes de Lucha con Agarre“. Revista de Educación Física, 59, 29-32. Nos basamos en la versión española del artículo.

9 Collinet (1994:29) asume la definición de algoritmo como un "conjunto de operaciones elementales que permiten resolver un problema".

10 E.g. Torres, 1989, 1990; Castarlenas, 1990; Brousse, 1993; Amador, 1995; Lima, 1997; Simon, 1997; Carratalà, 1998; Figueiredo, 1997, 1998; Molina \& Castarlenas, 2002; Espartero, Gutiérrez \& Villamón, 2003.

11 A este respecto, Prieto y cols. (2001) señalan que "por el mero hecho de que estas actividades [de lucha], en su dimensión social o 'popular', se entiendan de una determinada manera, no quiere decir que mediante el tratamiento didáctico que de ellas se realice no puedan transformarse en cooperativas o en medio de reflexión, ofreciéndose, por tanto, alternativas -así como críticas- a las visiones unilaterales de las mismas".

12 Por ejemplo: el atacante intenta romper distancias con desplazamientos, mientras el defensor intenta mantenerla, o el atacante intenta tocar las zonas corporales dejadas en "abierto" intencionalmente por el defensor (en una situación más evolucionada el defensor defiende y contra-ataca en las zonas "abiertas" del atacante primario).

13 Advertimos aquí que el tratamiento de determinadas habilidades técnicas, como luxaciones, estrangulaciones, o determinados tipos de golpeos, debería diferenciarse de las demás situaciones de combate, ya que aquí consideramos que los implicados han de poseer un dominio eficaz de los gestos de combate. Tal como señala Figueiredo (1998:V), "por razones de seguridad, deberán surgir después del diagnóstico de un nivel suficiente de madurez y de relaciones entre los alumnos para su abordaje".

\section{BIBLIOGRAFÍA}

Amador, F. (1995). "La enseñanza de los deportes de lucha". En Blázquez, D. (dir.). La Iniciación Deportiva y el Deporte Escolar. Barcelona: INDE, 351-368.

Amador, F. (1997). "Clasificación de los juegos y deportes de lucha". En VV.AA. El Judo y las Ciencias de la Educación Física y el Deporte. Vitoria/Gasteiz: Instituto Vasco de Educación Física, 5-22.

Araújo, D.; Volossovitch, A. (2005). "Fundamentos para o treino da tomada de decisão: uma aplicação ao andebol”. En Araújo, D. (dir.). O Contexto da Decisão: A acção Táctica no Desporto. Lisboa: Edições Visão e Contextos.

Bronchart, B. (1994). "Deportes de Lucha/Combate: los métodos activos". Revista de Educación Física, 59, 5-8.

Brousse, M. (1993). "Quel programme en sports d'opposition?". Revue E.PS, 242, 50-53.

Calmet, M. (2000). "Judo: la notion plurielle d'équilibre". En Terrisse, A. (dir.). Recherches en Sports de Combat et en Arts Martiaux - État des Lieux. Paris: Éditions Revue E.PS, 275-282.

Calmet, M.; Patinet, C. (1993). "Duos-Duels". Revue E.PS, 239, 64-67. 
Carratalà, V. (1998). "Los juegos y deportes de lucha en el marco de la Educación Física escolar”. En Villamón, M. (dir.). La Educación Física en el Currículum de Primaria. Valencia: Consellería de Cultura, Educació i Ciència, 269-316.

Castarlenas, J. (1990). "Deportes de Combate y Lucha: aproximación conceptual y pedagógica”. Apunts: Educació Física i Esports, 19, 21-28.

Castarlenas, J. (1993). "Estudio de las situaciones de oposición y competición. Aplicación de los Universales Ludomotores a los deportes de combate: el Judo". Apunts: Educació Física i Esports, 32, 54-64.

Collinet, S. (1992). "Algorithme et EPS. Un Exemple: Les Sports de Combat de Préhension". Revue E.PS, 237, 25-28.

Collinet, S. (1994). "Algoritmo y Educación Física-Deportiva. Un Ejemplo: los Deportes de Lucha con Agarre". Revista de Educación Física, 59, 29-32.

Espartero, J.; Gutiérrez, C.; Villamón, M. (2003). "Las Actividades de Lucha". En Tabernero, B. \& Márquez, S. (dir.). Educación Física: Propuestas para el cambio. Barcelona: Paidotribo, 283-324.

Lima, A. (1997). "Os Desportos de Combate (DC) como matéria de Educação Física (EF) nos Ensino Básico e Secundário". Horizonte, XV (86), 17-28.

Figueiredo, A. (1997). "Os Desportos de Combate nos Programas de Educação Física". Horizonte, XIV, (80), 36-39.

Figueiredo, A. (1998). "Os Desportos de Combate nas Aulas de Educação Física". Horizonte, XIV (81), Dossier.

Figueiredo, A. (2003). "Os exercícios de treino do Karate - Introdução à posição de base fundamental (guarda)". Karate Portugal, 3, 17-18.

Figueiredo, A. (2008). "The Combat Sports in Physical Education Classes - A Basic Perspective". En Cynarsky, W.J. (Ed.). Martial Arts and Combat Sports - Humanistic Outlook. Rzeszów: Wydawnictwo Uniwersytetu Rzeszowkiego, 145-149.

Griffin, L.; ButLeR, J. (2005). Teaching Games for Understanding - Theory, Researches and Practice. Chaimpaign: Human Kinetics.

Gutiérrez, C.; Pérez, M. (2009). "Study on scientific production in martial arts in Spain from 1990 to present”. En Cynarsky, W.J. (Ed.). Martial Arts and Combat Sports Humanistic Outlook. Rzeszów: Wydawnictwo Uniwersytetu Rzeszowkiego, 90-115.

López Ros, V.; CAstejón, F. (1998a). "Técnica, táctica individual y táctica colectiva: teoría de la implicación en el aprendizaje y la enseñanza deportiva (I)”. Revista de Educación Física, 68, 5-9.

López Ros, V.; Castejón, F. (1998b). "Técnica, táctica individual y táctica colectiva: implicación en el aprendizaje y la enseñanza deportiva (práctica) (II)”. Revista de Educación Física, 68, 12-16.

Molina, J.; Castarlenas, J. (2002). "Bases para una propuesta para la enseñanza del Judo en el contexto escolar”. En Castarlenas, J. \& Molina, J. (dir.). El Judo en la Educación Física Escolar. Barcelona: Hispano-Europea, 29-44.

Molina; J.; Villamón, M. (2000). "Principes stratégico-táctiques des sports de combate: l'exemple du judo". En Terrisse, A. (dir.). Recherches en Sports de Combat et en Arts Martiaux - État des Lieux. Paris : Éditions Revue EP.S, 175-184.

Olivera, J. (1984). "Prólogo". En Torres, G. 1000 Ejercicios y Juegos de Actividades de Lucha. Barcelona: Paidotribo, 6-8.

Parlebas, P. (1988): Elementos de Sociología del Deporte. Málaga: Unisport.

Parlebas, P. (2001). Juegos, Deporte y Sociedad. Léxico de Praxiología Motriz. Barcelona: Paidotribo.

La iniciación a los deportes de combate... $\diamond \quad$ Bruno Avelar \& Abel Figueiredo 
Prieto, J.; Gutiérrez, C.; Espartero, J.; Bricio, G.; Corujo, L.; Fernández, A. (2001). "El judo como actividad cooperativa. Algunas reflexiones en torno a una propuesta didáctica". Comunicación presentada en el I Congreso Estatal de Actividades Cooperativas, Junta de Castilla y León, Medina del Campo (Valladolid), 9 a de 12 de julio.

Ruiz Pérez, L.; Arruza, J. (2005). El Proceso de Toma de Decisiones en el Deporte - Clave de la Eficiencia y el Rendimiento Óptimo. Barcelona: Paidós.

Simon, H. (2003). "Las actividades de Lucha en el diseño curricular". En VV.AA. El Judo y las Ciencias de la Educación Física y el Deporte. Vitoria/Gasteiz: Instituto Vasco de Educación Física, 43-50.

Terrisse, A. (1991). "Pour un enseignement dialectique des sports de combat". Rerue EPS, 229, 23-26.

Terrisse, A. (1994). "Para una enseñanza 'Dialéctica”. Revista de Educación Física, 59, 9-14.

Terrisse, A. (1996). "Analyse de la transposition didactique du Judo: evolution du 'savoir combattre' dans l'enseignement du Judo à l'école à travers la Revue EPS de 1950 a 1993", Revue Française de Pédagogie, 116, 65-76.

Terrisse, A.; Quesada, Y.; Sauvegrain, J.; Hiegel, P. (1995). "Le Savoir Combattre: Essai d'Élucidation”. Revue EPS, 252, 26-29.

Theebom, S.; Knop, P. (1999). "Asian Martial Arts and approaches of instruction in Physical Education”. European Journal of Physical Education, 4, 146-161.

Torres, G. (1989). "Activitats de Lluita. Caracterització". Apunts: Educació Física $i$ Esports, 18, 71-74.

TORRes, G. (1990). "Las unidades motrices básicas luctatorias y su aplicación en la Educación Física”. Apunts: Educació Física i Esports, 24, 45-56.

Vergara, E. (2001). "Las actividades luctatorias y la formación en valores". Revista Educación Física y Deporte, 21 (2), 99-104. 